\title{
8. Ueber die Wärmeleitungsfähigkeit der Gase und ihre Abhängigkeit von der Temperatur (bei tiefen Temperaturen); von P. Adalbert Eckerlein.
} (Auszug aus der Münchener Dissertation.)

Ueber die Wärmeleitungsfähigkeit der Gase wurden in den letzten drei Jahrzehnten mehrfache experimentelle Untersuchungen angestellt. Die absoluten Wärmeleitungsconstanten stimmen noch wenig unter sich überein, und auch bezüglich der Abhängigkeit dieser Wärmeleitungsfähigkeit von der Temperatur haben die Versuche nock kein sicheres Resultat ergeben. $\left.{ }^{1}\right)$

Bei sämtlichen derartigen Versuchen geht Wärme auch durch Strahlung über, und es muss die Wärmestrahlung entweder direct berechnet oder durch "Differenzbeobachtungen" eliminirt werden. Es lag daher nahe, auch bei tiefen Temperaturen die experimentelle Untersuchung anzustellen. Bei Versuchen mit tiefen Temperaturen zur Ermittelung der Wärmeleitungsfähigkeit von Gasen ist nämlich besonders günstig der Umstand, dass nach unten hin der Anteil der ,Wärmeleitung“ relativ zum Anteil der Wärmestrahlung wächst, denn da die Wärmestrahlung sich ändert mit der vierten Potenz der absoluten Temperatur, nimmt sie sehr rasch mit der Temperatur ab, sodass immer mehr die „Leitung" zur Geltung kommt. Alle früheren Beobachtungsintervalle bei Ermittelung der Wärmeleitungsfähigkeit von Gasen haben den Eispunkt nicht überschritten, und vorliegende Untersuchung erstreckt sich zum ersten Male auf tiefe Temperaturen.

\section{\$ 1. Methode und Apparate.}

Da der Apparat nicht erhitzt werden durfte, wurde das von Winkelmann ${ }^{2}$ ) 1875 benutzte Verfahren gebraucht und

1) L. Boltzmann, Vorlesungen iiber Gastheorie. I. Teil. p. 88 . Leipzig 1896.

2) A. Winkelmann, Pogg. Ann. 156. p. 507. 1S75; Wied. Ann. 19. p. 656. 1883 . 
die Abkühlung eines und desselben Thermometers in zwei Hïllen mit verschiedenen Radien beobachtet. In die zwei Hüllen, deren Kugelradien

und

$$
R_{1}=1,641 \mathrm{~cm}
$$

$$
R_{2}=2,929 \mathrm{~cm}
$$

betrugen, passte mittels Schliff genau centrisch das Petrolätherthermometer.

Sein Gefäss, selbst kugelförmig, vom: inneren Radius

$$
r_{i}=0,4287 \mathrm{~cm}
$$

(er wurde vor der Füllung durch Wägung bestimmt), und vom äusseren Radius

$$
r_{a}=0,480 \mathrm{~cm} \text {, }
$$

war möglichst dünnwandig; die Länge des Thermometerstiels von der Kugel bis zum Schliff, also der Teil, welcher noch innerhalb der Hülle sich befand, wurde entsprechend dem Einfluss des Stiels auf die Abkühlung des Thermometers, auf ein Minimum reducirt und so gewählt, dass für die grössere Hülle zwischen Gefäss und Schliff gerade noch der nötige Raum verblieb, um in das cylindrische Rohr der Hülle das seitliche Ausflussrobr zur Luftpumpe und zu den Gasentwickelungsapparaten anzubringen. So betrug diese Länge bloss $3,7 \mathrm{~cm}$. Oberhalb des Schliffs begann sofort die Scala. Die Capillare wurde vor der Füllung mittels Quecksilberfaden calibrirt. Die Scala selber ist auf ein Glasrohr angebracht worden, welches über die Thermometersäule gestülpt durch ein drittes Glasrohr gedeckt war. Für die Ablesung des Einstellpunktes wurde ein Spiegel zur Vermeidung der Parallaxe benutzt. Die aufgetragene Millimeterteilung begann oben mit dem Punkt 0 ungefälı da, wohin der Eispunkt zu liegen kam, erstreckte sich nach oben bis zum Punkte 30, nach unten war sie $207 \mathrm{~mm}$ lang, sodass Punkt 207 gerade oberhalb des Schliffs zu liegen kam. Solche Wahl der Scala ermöglichte schon während der Messungen eine ungefähre Schätzung der abgelesenen Fadeneinstellung. Die Füllung des Thermometers wurde so ausprobirt, dass die Einstellung bei der Temperatur der flüssigen Luft möglichst nahe an den 
Schliff zu liegen kam, um den herausragenden Faden und seine Correctur, die hier bedeutend ins Gewicht fällt (vgl. \& 3), nicht unnötig zu vergrössern. Endlich sei noch bemerkt, dass das Thermometer beim Zuschmelzen des Capillarrohres in eine Kältemischung aus fester Kohlensäure und Chloroform von ca. $-70^{\circ}$ eingetaucht wurde. Dadurch war für alle höher gelegenen Temperaturen ein Ueberdruck in dem oberen Teil der Capillare erreicht, welcher dem Verdampfen und dem Ueberdestilliren der thermometrischen Flüssigkeit in diesen oberen Teil entgegentrat.

Petroläther als thermometrische Flüssigkeit für tiefe Temperaturen wurde zum ersten Male von Holborn und Wien ${ }^{1}$ ) und eingehender von Kohlrausch ${ }^{2}$ ) untersucht. Zur Messung absoluter Wärmebeträge ist die Kenntnis der Dichte und specifischen Wärme des Petroläthers, sowie der Aenderung dieser Grössen mit der Temperatur unentbehrlich. Abgesehen von einer Bestimmung der Dichte durch Kohlrausch, welcher für seine gebrauchte Fraction diese Grösse bei $17^{\circ}$ zu 0,6515 fand (l. c.), sowie mehrerer mittlerer Ausdehnungscoefficienten, liegen weitere Bestimmungen nicht vor.

§ 2. Dichte und specifische Wärme des Petroläthers und ihre Aenderung mit der Temperatur.

2. Die Dichte wurde bestimmt mit einer feinen, nach Mohr eingerichteten Waage. Ein Tauchkörper aus Glas, innen mit Quecksilber gefüllt, nach unten kegelförmig zugespitzt und $40,1548 \mathrm{~g}$ schwer, war mittels sehr dünnen Silberdrahtes an der einen Waagschale aufgehängt.

Bezeichnet man die wahre Dichte des Petroläthers für die Temperatur $t$ mit $d_{w, t}$ und die scheinbare mit $d_{s, t}$, so ist die Dichte von Petroläther bei $8^{0}$

$$
\text { 1. }\left\{\begin{array}{l}
d_{w, 8}=0,6408 \\
d_{s, 8}=0,6406
\end{array}\right.
$$

Unter der scheinbaren Dichte ist hier der Wert verstanden, welchen man erhält, wenn man die Contraction des Glases vernachlässigt; die wahre Dichte $^{3}$ ) ist hier wie im

1) M. Holborn u. Wien, Wied. Ann. 59. p. 26. 18926.

2) F. Kohlrausch, Wied. Ann. 60. p. 463-467. 1897.

3) Vgl. F. Kohlrausch, l. c. 
Folgenden unter der Berücksichtigung der Volumenänderung des Glases gefunden. Für Petroläther von $-77^{\circ}$ ist

$$
\text { 2. }\left\{\begin{array}{l}
d_{w(-77)}=0,6942 \\
d_{s(-77)}=0,6924 .
\end{array}\right.
$$

Für sehr tiefe Temperaturen versagte, wie man erwarten konnte, diese Methode vollständig, der Petroläther war zähe und hielt den Tauchkörper fest, sodass die Waage in jeder Lage blieb. Für die Messung der Dichte bei der Temperatur der flüssigen Luft wurde daher folgende Methode angewendet. Ein sehr dünnwandiges Glasrohr wurde aut die Form wie Fig. 1 gebracht. An die obere gezogene Capillare schloss sich die erste Erweiterung vom Volumen $V_{1}$ an, welche durch die folgende, gleichfalls gezogene Capillare mit der zweiten, grösseren Erweiterung vom Volumen $V_{2}$ in. Verbindung stand. $V_{1}$ und $V_{2}$ wurden beim Fertigen des Gefässes nach dem Augenmaass so gewählt, dass ungefähr das Verhältnis $V_{1} / V_{2}=\frac{1}{4}$ galt (vgl. Kohlrausch l. c.). ${ }^{1}$ )

Die zwei Capillaren wurden mit einer Scala versehen und das Gefäss so mit Petroläther gefüllt, dass

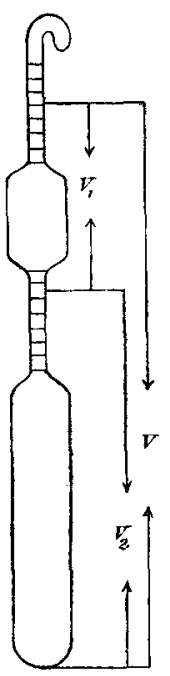

Fig. 1. die Einstellung seiner Niveaufläche in den Bereich der oberen Capillare zu liegen $\mathrm{kam}$ bei Zimmertemperatur, bei der Temperatur von flüssiger Luft jedoch in die zweite untere Scala fiel. Nach erreichter entsprechender Füllung wurde zugeschmolzen und die eigentlicheEinstellung beobachtet, einmal bei derZimmertemperatur, und dann in flüssiger Luft. Das Eintauchen in die flüssige Luft geschah nur sehr langsam und allmählich, um das Nachrücken der oberen Petrolätherschichten zu begünstigen und das Auftreten leerer Blasen zu verhüten. Nachdem die Einstellungspunkte möglichst genau bestimmt waren, wurden durch Wägung die Volumina, welche der Pe-

1 ) Dort heisst es nämlich, die Gesamtcontraction bei $-188^{\circ}$ war so gross, dass das Endvolumen $V_{-188}=\frac{4}{5} V_{0}$ betrug. Das entspricht den hier gewählten Verbältuissen, da $V_{2}=\frac{4}{5} V$ ist. 
troläther bei den zwei verschiedenen Temperaturen einnahm, bestimmt, und auf diese Weise ergab sich

$$
\text { 3. }\left\{\begin{array}{l}
d_{w(-183)}=0,8098 \\
d_{s(-183)}=0,8055
\end{array}\right.
$$

Aus den drei gefundenen Werten, welche sich auf verhältnismässig weit auseinander liegende Temperaturen beziehen, berechnet sich allgemein für tiefe Temperaturen

I. die wahre Dichte des Petroläthers zu

$$
d_{w, t}=0,6444-0,000472 t+0,0000024 t^{2} ;
$$

II. die scheinbare Dichte zu

$$
d_{s, t}=0,6440-0,000442 t+0,0000023 t^{2} .
$$

3. Die folgenden Bestimmungen der specifischen Wärme des Petroläthers sind nach der Mischungsmethode ausgeführt. Da auch die specifische Wärme des Glases für die Berechnung des Wasserwertes vom Thermometergefäss bekannt sein muss, Bestimmungen für Temperaturen unter $0^{0}$ aber noch nicht vorliegen, wurde sie auch für das zur Anfertigung des Thermometers verwendete Glas (Normalglas) nach derselben Methode bestimmt. Arbeiten auf diesem Gebiet bei tiefen Temperaturen liegen erst seit der neuesten Zeit vor. $B e h n^{1}$ ) hat Metalle nach der Mischungsmethode in Bezug auf ihre specifische Wärme bei tiefen Temperaturen (flüssige Luft, Kältemischung aus Kohlensäure und Aether) untersucht. Von seiner Methode habe ich erst Kenntnis erhalten, nachdem diese Untersuchungen schon vollendet waren.

Es standen mir für diese Versuche äusserst feine und empfindliche Quecksilberthermometer zur Verfügung. Das im Calurimeter benutzte Thermometer umfasste eine Scala von $0^{\circ}$ bis $+11^{\circ}, 1$ Grad war ca. $25 \mathrm{~mm}$ lang und in 50 Teile geteilt, und liess Ablesungen bis auf $1 / 100^{\circ}$ mit dem Fernrohr bequem zu. Als thermometrische Flüssigkeit benutzte ich nicht Wasser, wie Behn in seinen Untersuchungen anwendet, sondern rectificirtes Terpentinöl.

1) U. Behn, Wied. Anu. 66. p. 237. 1898; Ann. d. Phys. 1. p. 257.1900 . 
Da Terpentinöl, auch rectificirtes, nicht säurefrei ist, musste das Calorimeter, ein dünnwandiges Gefäss aus Messing, inwendig vergoldet werden, desgleichen der gebrauchte Messingrührer, welcher durch einen kleinen Motor bewegt wurde. Da der Petroläther selber für diese Untersuchungen in Messing eingeschlossen wurde, fiel auch Messing unter diese Bestim. mungen. Es gelang nämlich unter grosser Vorsicht, den Petroläther in eine metallische Hülle vollständig dicht zu verschliessen. Eine Kugel aus Messing war mit einem Hals versehen, in welchen eine Versenkschraube gut eingepasst war. Die Kugel wurde mit Petroläther gefüllt, die Schraube sofort eingedreht und ihr Ende mit dem Hals an der Kugel verlötet. Die Kugel war dabei in einem Eisbad, sodass nur der Ansatz herausragte. Vor der Verlötung wurde die Kugel noch rasch auf die Waage gelegt, um das Gewicht des eingeschlossenen Petroläthers absolut zu bestimmen. Dass vollkommen dichter Abschluss hergestelit wurde, verbürgt der Umstand, dass das Gewicht dieser Kugel nach einem balben Jahr um nichts abgenommen hatte.

Die drei zu untersuchenden Körper: der Glaskörper von cylindrischer Form, der Messingkörper, aus einer dickeren Platte geschnitten vom selben Material wie die Hülle des Petroläthers und vergoldet, die Kugel (gleichfalls vergoldet) mit dem Petroläther, wurden bei den Versuchen in einem durch Kältemischungen erniedrigten Terpentinölbad auf die gewünschte Anfangstemperatur gebracht. Bei der Temperatur der flüssigen Luft' wurde diese selber als Kältebad gebraucht. War die Temperatur in diesen angewendeten Mischungen constant geworden, so wurde in einem bestimmten Zeitmoment von einem Gehülfen der Körper möglichst rasch in das Calorimeter gebracht (die Zeitdauer dazu betrug Bruchteile von oder höchstens $1 \mathrm{sec}$ ) und dann die Abkühlung von 20 zu 20 Secunden mit Fernrohr abgelesen. Die Menge des Terpentinöls, welche haften blieb, wenn der Körper dem Bad entnommen und in das Calorimeter übertragen wurde, war bestimmt worden für die zwei Kältemischungen Eis und Salz, Kohlensäureschnee und Aether, und zwar durch viele Wägungen, aus welchen das Mittel zur Anwendung kam (vgl. Tab. I, wo die Zahlenwerte angegeben sind); bei der Tem- 
peratur der flüssigen Luft fiel diese Correctur weg. Teilchen von flüssiger Luft blieben an den Körpern kaum haften, und wenn wirklich, dann hat die während des Transportes ins Calorimeter aus der Umgebung zugeführte, sehr kleine Wärmemenge leicht das Verdampfen dieser Teilchen bewirken können, weil flüssige Luft eine äusserst geringe Verdampfungswärme besitzt. Der Einfluss der Wärmestrahlung aus der Atmosphäre auf das Calorimeter wurde in der üblichen Weise vor und nach der Temperaturausgleichung beobachtet und in Rechnung gebracht.

Für die specifische Wärme des Terpentinöls wurde der Wert nach Regnault zu

$$
c_{t}=0,41058+0,0012386 t-0,000003981 t^{2} \text { l) }
$$

genommen. Uebrigens habe ich die mittlere specifische Wärme zwischen 4 und $9^{\circ}$ selber bestimmt zu 0,4187 , ein Wert, welcher sehr gut stimmt mit dem aus der Regnault'schen Formel berechneten (0,41851). Der Wasserwert des Calorimeters, Rührers und Thermometers wurde für die verschiedenen auftretenden Mitteltemperaturen genau berechnet. Auch für das Terpentinöl im Calorimeter wurde die jeweilige mittlere specifische Wärme genommen. Von ca. 40 Versuchen wurden die besten zur Berechnung benutzt. Was die Versuche geben, sind nur mittlere specifische Wärmen $c_{m}$.

Bevor ich die Werte der $c_{t}$ angebe, mögen die zu Grunde liegenden gemessenen Grössen hier gedrängten Platz finden (vgl. Tab. I).

Es werden die wahren specifischen Wärmen $c_{t}$ aus diesen gefundenen $c_{m}$ :

für Glas: $\quad c_{t}=0,1614+0,000763 t+0,00000294 t^{2}$;

für Messing: $\quad c_{t}=0,0890+0,000316 t+0,00000103 t^{2}$;

Pür Petroläther: $c_{t}=0,4194-0,000395 t-0,00000143 t^{2}$;

gültig im Intervall von ca. $+10^{\circ}$ bis $-190^{\circ}$.

Es fällt hier einigermaassen auf, dass die specifische Wärme des Petroläthers mit abnehmender Temperatur zu-

1) A. Wüllner, Lehrb. der Experimentalphysik 2. p. 586. 1896. 
Wärmeleitungsfähigkeit der Gase.

\begin{tabular}{|c|c|c|c|c|c|}
\hline \multirow{2}{*}{\multicolumn{2}{|c|}{ 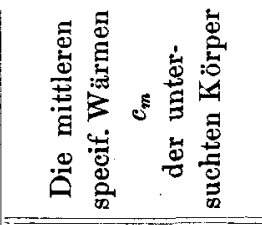 }} & \multirow{2}{*}{ 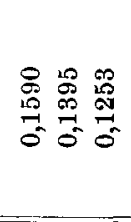 } & \multirow{2}{*}{ 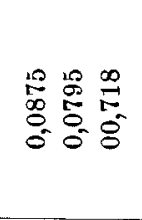 } & \multicolumn{2}{|c|}{ 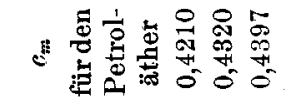 } \\
\hline & & & &  & 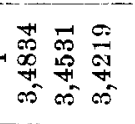 \\
\hline \multicolumn{2}{|c|}{  } & $\frac{\infty}{=} \underset{0}{=} 1$ & $\begin{array}{l}0 \\
\stackrel{0}{0} \\
0 \\
0\end{array}$ & & $\stackrel{\infty}{N}_{0}^{\infty} 1$ \\
\hline \multirow{2}{*}{ 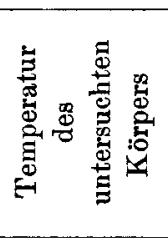 } & 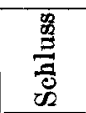 & 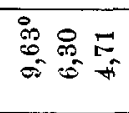 & 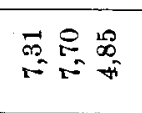 & & 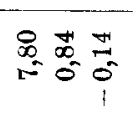 \\
\hline & 它 & $\begin{array}{lll}8 & 0 & \infty \\
5 & \infty & 0 \\
\infty & \infty & 10 \\
1 & \infty & \infty \\
1 & 1 & 1\end{array}$ & $\begin{array}{lll}0 & 0 & \infty \\
20 & 15 & 9 \\
0 & 0 & 0 \\
0 & 15 & 0 \\
1 & 1 & 1\end{array}$ & & $\begin{array}{lll}0 & 0 & \infty \\
N & 0 & 0 \\
0 & 0 \\
0 & 0 \\
0 & \infty \\
1 & 1 & 1\end{array}$ \\
\hline \multicolumn{2}{|c|}{ 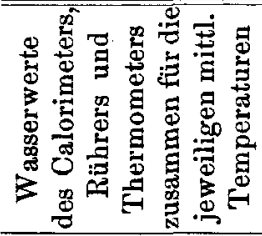 } & 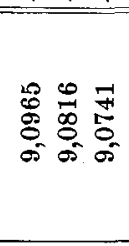 &  & & 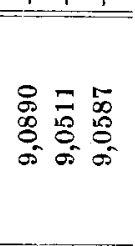 \\
\hline \multicolumn{2}{|c|}{  } & 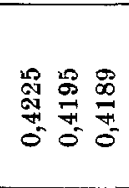 & 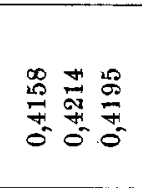 & & 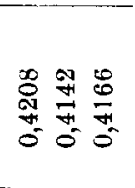 \\
\hline \multirow{2}{*}{ 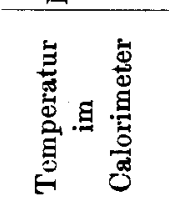 } & 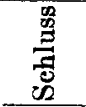 & $\begin{array}{l}i_{0} 0 \\
0 \\
0 \\
0\end{array}$ & $\begin{array}{l}\overrightarrow{0} \\
\mathbf{p}_{1}\end{array}$ & & $\stackrel{\infty}{\infty} \stackrel{\infty}{\infty} \frac{\pi}{0}$ \\
\hline & 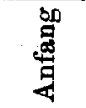 & 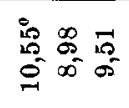 & 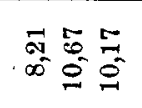 & & 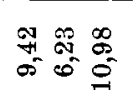 \\
\hline 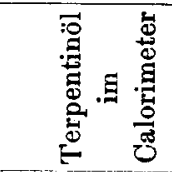 & 80 & 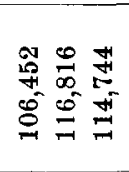 & 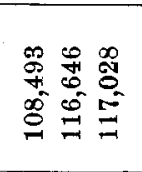 & & 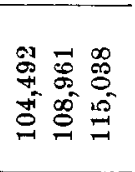 \\
\hline 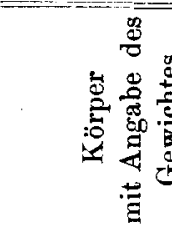 & & 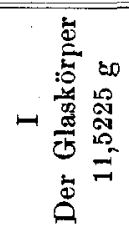 & 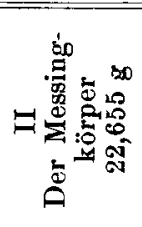 & 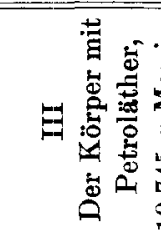 & 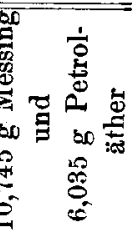 \\
\hline
\end{tabular}


nimmt. Diese Zunahme mit fallender Temperatur, welche dieses Destillationsproduct mit dem Quecksilber zufällig gemeinsam zu haben scheint, machte sich bei allen Versuchen, die angestellt wurden, gleich bemerkbar. Schon im Intervall von $0^{\circ}$ bis $-80^{\circ}$ ergiebt sich dies, sodass man für die Erklärung dieses Ergebnisses kaum Erstarrung des Petroläthers annehmen darf; eine ganz befriedigende Aufklärung aber giebt eine Abhandlung von L. Sohncke ${ }^{1}$ ) ,Ueber die Aenderung der specifischen Wärme mit der Temperatur". Die daselbst definirte „äussere Disgregationsarbeit", auf das Verhalten des Petroläthers angewendet, würde aussagen:

„In höheren Temperaturen sind wegen der eingetretenen enormen Volumenvergrösserung des Petroläthers die Molecüle weiter auseinander gerïckt als zuvor, diese wirken daher alsdann mit geringeren Kräften aufeinander als bei tieferen und tiefsten Temperaturen: Dieses Verhalten der Moleküle bedingt daher bei tiefen Temperaturen den Aufwand einer grösseren äusseren Disgregationsarbeit als bei höheren Temperaturen und die Zunahme dieser Arbeit bedingt hinwiederum die Zuführung einer grösseren Wärmemenge, d. h. eine Zunahme der specifischen Wärme mit sinkender Temperatur."

\section{\$3. Die Temperaturmessung.}

Sämtliche Temperaturen unter $0^{0}$ sind angegeben und genommen von einem Thermoelement Eisen-Constantan, welches laut Prüfungsschein vom 15. Januar 1898 in der PhysikalischTechnischen Reichsanstalt auf seine elektromotorische Kraft bei tiefen Temperaturen bis $2 u-77,2^{\circ}\left(-80^{\circ}\right)$ untersucht wurde, und einem Spannungsmesser ,Keiser \& Schmidt Nr. 1140", der gleichfalls mit Prüfungsschein aus der Physikalisch-Technischen Reichsanstalt (15. Januar 1898) versehen ist. Um die noch tiefer gelegenen Temperaturen mit mehr Sicherheit zu bestimmen, als diese Hülfsmittel erlauben, wurde im hiesigen $v$. Linde'schen Kältelaboratorium die elektromotorische Kraft des benutzten Thermoelementes für die Tem-

1) L. Sohncke, Wied. Ann. 66. p. 111. 1898. 
peratur $-188^{\circ}$ durch die Güte der Herren Dr. Sieder und Dr. Linde bestimmt. Für die Thermometerscala wurden folgende drei grundlegende Punkte der wahren Scala wiederholt bestimmt und dabei folgende Einstellungen gefunden:

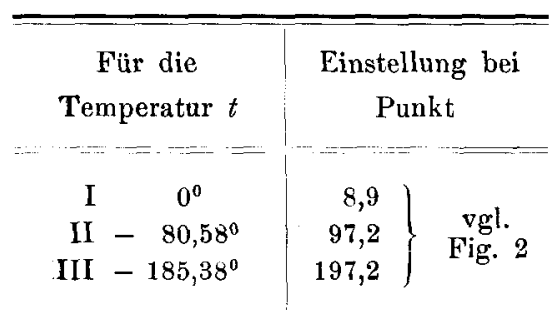

Bei der Fixirung dieser drei Punkte tauchte die ganze Scala in das Temperaturbad ein, dessen Temperatur mit dem Thermoelement gemessen wurde.

Setzt man, um auf einen $\mathrm{Zu}$ sammenhang $z$ wischen Temperatar und Flüssigkeitssäule zu kommen, den Punkt III von flüssiger Luft für die Rechnung gleich $0^{0}$ und zählt von da nach oben die Grade positiv, so entspricht den obigen Angaben, wenn $t$ die neu eingeführte Temperaturangabe, $l_{t}$ die entsprechende Länge des Fadens ab Punkt III bedeutet:

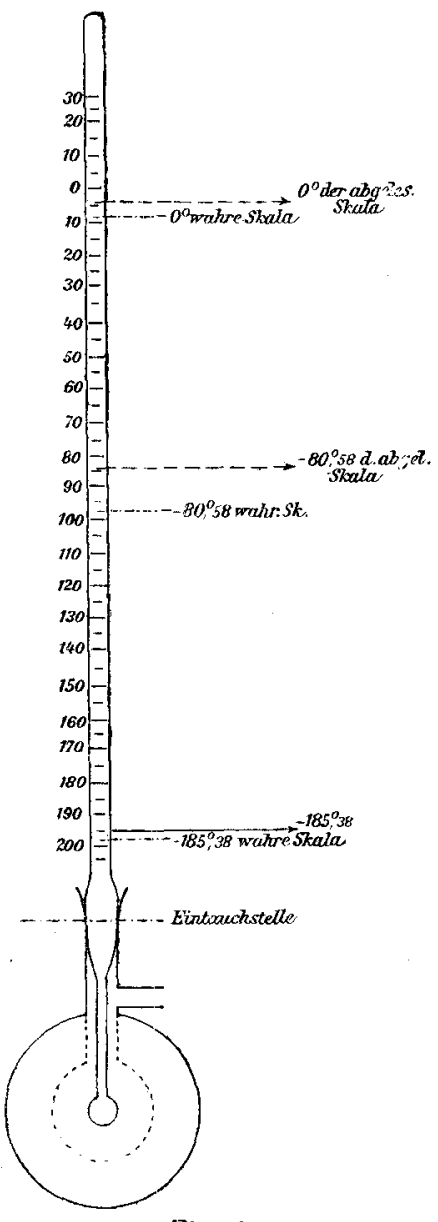

Fig. 2.

\begin{tabular}{r|c|c}
\hline Punkt & $\begin{array}{c}\text { hat die } \\
\text { Temperatur } t\end{array}$ & $\begin{array}{c}\text { und die zugehörige } \\
\text { Länge } l_{t}\end{array}$ \\
\hline I & $185,38^{\circ}$ & $188,3 \mathrm{~mm}$ \\
II & $104,8^{\circ}$ & $100, "$, \\
III & $0^{\circ}$ & $0, "$,
\end{tabular}

Annalen der Physik. IV. Folge. 3. 
Nimmt man als Beziehung zwischen $t$ und $l_{t}$ :

$$
\frac{t}{l_{t}}=\alpha+\beta t,
$$

so wird

$$
\begin{aligned}
& \alpha=1,130593 \\
& \beta=-0,0007881
\end{aligned}
$$

und es ist $t$ gegeben durch

$$
t=\frac{1,130593 l_{t}}{1+0,0007881 l_{t}} .
$$

Nach dieser Formel wurde die Scala berechnet. ${ }^{1}$ )

Bei den Versuchen wurde die wahre Scala nie abgelesen, weil immer die ganze Thermometerscala mit der Teilung aus dem auf seine Temperatur zu messenden Raume herausragte, mithin eine andere Temperatur hatte, als der unter der Scala liegende Teil; es handelte sich also, kurz gesagt, um ,die Correctur für den herausragenden Faden".

Die Bestimmung der Einstellungspunkte auf der abgelesenen Scala (d. h. mit herausragendem Faden) für die drei angegebenen Fundamentaltemperaturen, wobei also die Eintauchstelle des Thermometers so gewählt war, wie es bei den Versuchen zutrifft (vgl. Fig. 2), wurde mit möglichster Sorgfalt und wiederholt vorgenommen, und hatte als Ergebnis:

\begin{tabular}{c|c}
\hline $\begin{array}{c}\text { Für die } \\
\text { Temperatur } \\
t\end{array}$ & $\begin{array}{c}\text { giebt die abge- } \\
\text { lesene Scala die } \\
\text { Einstellung }\end{array}$ \\
\hline \hline I $0^{\circ}$ & $\begin{array}{c}4,5 \\
\text { II }-80,58\end{array}$ \\
III $-185,38$ & $\left.\begin{array}{c}84,5 \\
195\end{array}\right\}$ vgl. Fig. 2
\end{tabular}

Vergleicht man diese Einstellungen der abgelesenen Scala mit denen der wahren Scala, so findet man selbstrerständlich die Einstellungspunkte der abgelesenen Scala höher gelegen

1) Vgl. näbere Angaben in der Dissertationsschrift. 
als die der wahren, und die beträchtlichen Differenzen der Flüssigkeitssäulen sỉnd

$$
\begin{aligned}
& \text { für Punkt I: } 4,4 \mathrm{~mm} \\
& \text {, , II: } 12,7 \mathrm{~mm} \\
& \text {, , III: } 2,2 \mathrm{~mm} \text {. }
\end{aligned}
$$

Bezeichnet man die Flüssigkeitshöhen der abgelesenen Scala mit $l$, die Differenz, welche von diesen $l$ weggenommen werden muss, um auf die Flüssigkeitshöhen der wahren Scala zu kommen, mit $y$, so lässt sich auf Grund der drei bekannten Differenzen zu jedem $l$ das zugehörige $y$ finden, vermöge der Formel:

$$
y=0,080995 l+0,000747994 l^{2}-0,0000048152 l^{3}
$$

und so die ahgelesene Scala auf die wahre reduciren.

Nach den angeführten Grössen wurde die Scala berechnet; nähere Angaben vgl. die Dissertationsschrift, besonders Tab. II p. 24.

\section{\$ 4. Die Temperaturbäder.}

Constante Temperaturbäder für tiefe Temperaturen sind leider nur spärlich vorhanden. In der Nähe von $-80^{\circ}$ haben Cailletet und Colardeau ${ }^{1}$ ) im Jahre 1888 mehrere Kältemischungen mit fester Kohlensäure hergestellt und benutzt. Von den (l. c.) erwähnten Mischungen wurde die gebräuchlichste genommen: Aether und Kohlensäureschnee.

Sehr constante und sehr tiefe Temperaturbäder liefert die flüssige Luft.

1. Bei der Aetherkohlensäuremischung wurden die Erfahrungen von Walther Hempel ${ }^{2}$ ) benutzt. Nur die zwei wesentlichsten Fragen mögen hier behandelt werden: Wie erzielt man die beste Wirkung der Kältemischung? Wie erreicht man die tiefste Temperatur und verlässigste Constanz, und wie verhält sich das Kältebad, wenn ein wärmerer Körper hineingebracht wird?

1) L. Cailletet und E. Colardeau, Compt. rend. 106. p. 1631 u. ff. 1888 .

2) Walther Hempel, Ueber die Arbeiten bei niederen Temperaturen. Ber. d. Deutschen chem. Gesellsch. 31. (III.) p. 2993-2997. 1898. 
Die tiefste Temperatur, welche ich mit dieser Kältemischung erreicht habe, trat dann ein, wenn die gemischte Masse mit Kohlensäure ganz gesättigt, kurz ein förmlicher Teig war. Wenn ich diese äusserste Temperatur in der Kältemischung noch nicht erreicht hatte, habe ich solange Kohlensäure beigemengt, bis sie eintrat, auf diese Weise war der Nachteil bei den Versuchen, im Abkühlungsbad verschiedene Temperaturen zu haben, am einfachsten $z u$ beseitigen. Diese unterste Temperatur der Kohlensäureäthermischung, mit dem Thermoelement unter stetigem Rühren des Gemisches gemessen, war $-80,58^{\circ}$. Brachte man einen Körper aus der Temperatur der Umgebung in das Kältebad, so begann die Kohlensäure zwar lebendiger zu kochen und zu perlen, aber eine Temperaturerhöhung konnte nicht beobachtet werden. Um dies eingehender zu prüfen, wurde z. B. einer Kugel aus dünnem Glas und mit einem Stiel, ganz ähnlich mit den Hüllen der Thermometer, von oben her durch den Stiel Wärme zugeführt, während sie in die Kältemischung tauchte. Eine Temperaturerhöhung wurde auch da nicht bemerkt.

2. Flüssige Luft lieferte das zweite, angewendete Kältebad. Dass sie sehr gut brauchbare, constante Temperaturbäder giebt, fanden frühere schon, wie Holborn und Wien ${ }^{1}$ ), Kohlrausch etc. Allerdings langsam mit der Zeit ändert sie ihre Temperatur (l. c. p. 217). Wie ich die flüssige Luft verwendete, war sie bezüglich ihrer Temperatur wohl constant während der Versuchsreihen, nicht aber, wie sich erwarten lässt, in den zeitlich verschiedenen Lieferungen, die ich erhielt; da hatte sie bald $-188^{\circ}$, bald $-185^{\circ}$ oder $-182^{\circ}$. Doch war die Temperatur mit einer einzigen Ausnahme (vgl. Tab. V) während aller Abkühlungsversuche mit dem Petrolätherapparat nahezu dieselbe, und zwar mit dem Thermoelement gemessen: $-185,38^{\circ}$ (entsprach der elektromotorischen Kraft $7220 \mu$-Volt). In der bezeichneten Tabelle finden sich drei Versuche mit der Temperatur $-187,65^{\circ}$ im Abkühlungsbad. Wie schwer diese Temperaturdifferenz ins Gewicht fällt,

1) I. Holborn und M. Wien, Wied. Ann. 59. p. 217. 1896 ; vgl. auch F. Wroblewski, Wied. Ann. 25. p. 371.1885 und 26. p. 134. 1885; sowie Kohlrausch, Wied. Ann. 60. p. 464. 1897. 
zeigt ein Vergleich der Abkühlungszeiten (Tab. V). Kleine Abweichungen (bis zu $20 \mu$ Volt) durften vernachlässigt werden. Als Behälter für die flüssige Luft dienten dünne, dicht mit Wolle umgebene Glasgefässe. Un während der Versuche immer dieselbe Niveaufläche im Kältebad der flüssigen Luft zu haben, musste das nötige Quantum immer nachgegossen werden, wenn ein Abnehmen der flüssigen Luft durch Verdampfen wahrgenommen werden konnte, die Temperatur im Kältebade wurde während der Abkühlungsversuche immer gemessen, beides musste natürlich durch einen Gehülfen geschehen.

\section{§ 5. Die Berechnung der Versuche. ${ }^{1}$ )}

1. Bezeichnet $k_{t}$ die Wärmeleitungsfähigkeit eines Gases für eine $z \mathfrak{u}$ bestimmende Mitteltemperatur $t$, dann $v_{t}$ die $\mathrm{Ab}$ kühlungsgeschwindigkeit des Thermometers in der Hülle, $C$ den Wasserwert des Thermometers bei dieser Temperatur $t$, $\sigma$ die Strahlungsconstante und $g_{1}$ wie $g_{2}$ Correctionsglieder, so dienen zur Berechnung von $k$ die zwei Gleichungen:

$$
C_{t} \cdot v_{1}=k_{t} \frac{4 \pi R_{1} r}{R_{1}-r}+\sigma \cdot 4 \pi r^{2}+g_{1}
$$

und

$$
C_{t} \cdot v_{2}=k_{t} \frac{4 \pi R_{2} r}{R_{2}-\mathrm{r}}+\sigma .4 \pi r^{3}+g_{2} .
$$

Dabei bedeutet $v_{1}$ die Abkühlungsgeschwindigkeit für die Hülle mit dem (kleineren) Radius $R_{1}, v_{2}$ die nämliche Grösse für die Hülle vom (grösseren) Radius $R_{2}$; von den Gliedern rechts in den zwei Gleichungen beziehen sich je die ersten auf den durch "Leitung" übertragenen Wärmeteil und zeigen die Abhängigkeit dieses Anteiles vom Radius der Hülle, die zweiten Glieder, ihrem Werte nach gleich, geben den Anteil der Strahlung und lassen diese, wie es der Fall ist, als unabhängig erkennen von der Hülle, die Correctionsgrössen $g_{1}$ und $g_{2}$ endlich beziehen sich auf den Stiel des Thermometers innerhalb der Hülle. Von ihnen, wie überhaupt von der Einwirkung des Stieles soll später noch die Rede sein; für jetzt

1) Ueber die Versuchsanordnung; vgl. Dissertationsschrift p. 27. 
sei nur bemerkt, bei der Bestimmung des $k$ aus den zwei Gleichungen heben sich die zwei Correctionsgrössen zum guten Teil auf. Setzt man nun in erster Annäherung $g_{1}-g_{2}=0$, dann erhält man zwei Gleichungen für die Wärmeleitungsconstante:

$$
k_{t}=\frac{C_{t}\left(R_{1}-r\right)\left(R_{2}-r\right)\left(v_{1}-v_{2}\right)}{4 \pi r^{2}\left(R_{2}-R_{1}\right)} .
$$

2. Die Abkühlungsgeschwindigkeit $v$ wurde berechnet nach Winkelmann ${ }^{1}$ ); er bestimmte die v nach dem Newton'schen Abkühlungsgesetz durch

$$
v=\log \left\{\frac{t_{0}-y}{t_{1}-9}\right\} \frac{1}{\log e} \frac{1}{T_{1}},
$$

wo hier $\vartheta$ die Temperatur der Hülle oder des Bades, $T_{1}$ die Zeit und $t_{0}$ die Temperatur des Thermometers ist zur Zeit 0.

Oder wenn ich die Temperatur von der Temperatur des Bades an, diese gleich Null gesetzt, rechne und mit $\vartheta$ die Zeit bezeichne:

$$
v=\log \left(\frac{t_{0}}{t_{1}}\right) \frac{1}{\log e} \frac{1}{\vartheta_{1}} .
$$

Aus der Beobachtungsreihe der $t$ und $\vartheta$ berechnete Winkelmann nach seinem ersten Verfahren den Mittelwert der $v$ und bestimmte hernach nach einem (l. c.) angegebenen Verfahren, auf welche Mitteltemperatur dieser Wert sich beziehe. Auf diese Weise war die Frage nach der Abhängigkeit der Grösse $v$ von der Temperatur sehr einfach gelöst. Es gehörte also zum Werte

die Temperatur

$$
v_{t}=\frac{v_{1}+v_{2}+v_{8}+\ldots+n_{n}}{n}
$$

$$
t=\frac{n \cdot t_{0}+t_{1}+t_{2}+\ldots+t_{n}}{4 n} .
$$

Diese Methode hat dann Winkelmann ${ }^{2}$ ) im Jahre 1880 verbessert, in dieser verbesserten Methode giebt er in der Berechnung der mittleren Abkühlungsgeschwindigkeit den ein-

1) A. Winkelmann, Pogg. Ann. 157. p. 514-517. 1876.

2) A. Winkelmann, Wied. Ann. 11. p. 480 u. $481,1880$. 
zelnen Werten $v_{1}, v_{2}, v_{3} \ldots$ die Gewichte der dazugehörigen Abkühlungszeiten $t_{1}, t_{2}, t_{3} \ldots$ deshalb, weil bei dem bloss einfach arithmetischen Mittel die bei der Beobachtung der kleineren Abkühlungszeiten auftretenden Beobachtungsfehler um vieles schwerer ins Gewicht fallen, als die bei der Messung grösserer Abkühlungszeiten begangenen. Es wäre also nach der verbesserten Winkelmann'schen Berechnungsweise, wenn $\vartheta$ die Zeit, $t$ die Temperatur bedeutet:

$$
v_{t}=\frac{v_{1} \vartheta_{1}+v_{2} \vartheta_{2}+v_{3} \vartheta_{3}+\ldots+v_{n} \vartheta_{n}}{\vartheta_{1}+\vartheta_{2}+\vartheta_{3}+\ldots+\vartheta_{n}}
$$

und analog die entsprechende Temperatur:

$$
t=\frac{t_{0}}{4}+\frac{t_{1} \vartheta_{1}+t_{2} \vartheta_{2}+t_{3} \vartheta_{3}+\ldots+t_{n} \vartheta_{n}}{4\left(\vartheta_{1}+\vartheta_{2}+\vartheta_{3}+\ldots+\vartheta_{n}\right)} .
$$

Zur Berechnung dieses $v_{t}$ ist es durchaus nicht notwendig, erst die $v_{1}, v_{2}, v_{3} \ldots$ einzeln $z u$ bestimmen und sie dann mit den zugehörigen Abkühlungszeiten zu multipliciren, denn da

so ist einfach

$$
v_{n} \vartheta_{n}=\frac{1}{\log e}\left\{\log t_{0}-\log t_{n}\right\}
$$

$$
\begin{gathered}
v_{t}=\frac{n \cdot \log t_{0}-\sum\left(\log t_{v}\right)}{\log e \cdot \sum\left(\vartheta_{\nu}\right)} \\
t=\frac{t_{0}}{4}+\frac{\sum\left(t_{\nu} \cdot \vartheta_{v}\right)}{4 \sum\left(\vartheta_{\nu}\right)} .
\end{gathered}
$$

Die einzelnen Werte $v_{1}, v_{2}, v_{3} \ldots$ habe ich, obwohl für den Mittelwert $v_{t}$ nicht gefordert, dennoch ausgerechnet und in den Tabellen angeführt, weil man an ihnen die Aenderung mit der Temperatur verfolgen kann. Es zeigen diese Werte $v \log e$ ein langsames, stetiges Abnehmen mit ganz wenigen Ausnahmen, welchen die Bedeutung von Beobachtungsfehlern zukommt. Die mittlere Temperatur $t$, auf welche das $v_{t}$ sich bezieht, ist auch bei den einzelnen Tabellen bemerkt.

Die Mittel der Abkühlungszeiten sind auf zweifache Weise berechnet; das eine Mal sind es die arithmetischen Mittel der beobachteten Grössen schlechtweg, das andere Mal sind sie bestimmt worden auf folgende Weise: 
Ist $0, \vartheta_{1}, \vartheta_{2}, \vartheta_{3} \ldots \vartheta_{n}$ die Reihe der notirten Abkühlungszeiten, dann $t_{0}, t_{1}, t_{2}, t_{3} \ldots t_{n}$ die Reihe der abgelesenen Temperaturen, so gilt: es kühlt sich das Thermometer ab

$$
\begin{aligned}
& \text { um }\left(t_{0}-t_{1}\right)^{0} \text { in } \vartheta_{1} \mathrm{sec}, \\
& "\left(t_{1}-t_{2}\right)^{0}, \quad\left(\vartheta_{2}-t_{1}\right) \mathrm{sec}, \\
& "\left(t_{2}-t_{3}\right)^{0}, \quad\left(\vartheta_{3}-\vartheta_{2}\right) \text { sec, } \\
& \cdot \cdot \cdot \cdot \cdot \cdot \cdot \cdot \cdot \cdot \cdot \cdot \cdot \cdot \\
& \operatorname{um}\left(t_{n-1}-t_{n}\right)^{0} \text { in }\left(\theta_{n}-\vartheta_{n-1}\right) \text { sec. }
\end{aligned}
$$

Die Differenzen $\vartheta_{1},\left(\vartheta_{2}-\vartheta_{1}\right),\left(\vartheta_{3}-\vartheta_{2}\right), \ldots\left(\vartheta_{n}-\vartheta_{n-1}\right)$ wurden für die einzelnen Versuche berechnet, aus den Werten dieser Differenzen wurde das arithmetische Mittel genommen, d. h. also das Mittel aus allen $\vartheta_{1}$ sowie das Mittel aus allen $\left(\vartheta_{2}-\vartheta_{1}\right)$ etc. und aus diesen Mittelwerten wurden mittlere $\vartheta_{1}, \vartheta_{2}, \vartheta_{3} \ldots \vartheta_{n}$ berechnet. $^{1}$ ) Auf diese Art werden die Beobachtungsfehler möglichst verteilt und insbesondere verteilt auf das Intervall, wo sie entstanden, und sie übertragen sich nicht so sehr auf die folgenden Intervalle. Versuche mit Drucken, bei denen auch die Strömungen noch Einfluss haben, werden in den Tabellen dennoch angeführt, um auch in die Wirkung der Strömungen bei tiefen Temperaturen einen Einblick zu gewinnen.

3. Der Temperaturcoefficient $\gamma$ ergiebt sich aus:

$$
\frac{k_{T}}{k_{t}}=\frac{k_{0}(1+\gamma \cdot T)}{k_{0}(1+\gamma \cdot t)}=\frac{C_{T} \cdot\left(V_{1}-V_{2}\right)}{C_{t}\left(v_{1}-r_{2}\right)} \cdots
$$

Dabei sind $C_{T}, V_{1}$ und $V_{2}$ die auf $T$ sich beziehenden, schon definirten Grössen, $C_{t}, v_{1}$ und $v_{2}$ gehören zu $t$. Man sieht, dass die Dimensionen der Apparate bei der Berechnung der $\gamma$ in Wegfall kommen, $\gamma$ aber abhängig ist vom Quotienten der Wasserwerte bei den verschiedenen Temperaturen.

Noch sind die Wasserwerte $C_{t}$ und $C_{T}$ anzugeben, welche sich auf die Mitteltemperaturen beziehen.

Es ist:

$$
\begin{aligned}
t & =-59^{\circ} \\
T & =-149,5^{\circ} .
\end{aligned}
$$

1) Zum Unterschied von dem gewöhnlichen Mittel sind die Columnen, welche die so berechneten Mittel enthalten, oben mit einem Stern* bezeichnet. 
Dazu gehören:

$$
\begin{aligned}
C_{t} & =0,143 \overline{\mathrm{s}}, \\
C_{T} & =0,1528 .
\end{aligned}
$$

Bei der Berechnung dieser Wasserwerte des Petrolätherthermometers (bez. seines Gefässes) wurde nicht nur auf die Aenderung der specifischen Wärme des Petroläthers und des Glases mit der Temperatur Rücksicht genommen, sondern auch auf die Abhängigkeit der Dichte des Petroläthers von der Temperatur, welche hier nicht vernachlässigt werden kann. Es liegen dabei die Resulate von $\$ 3$ dieser Arbeit zu Grunde.

I. Bestimmung der Leitungsfähigkeit $k$ für atmosphärische Luft und des Temperaturcoefficienten $\gamma$.

Die atmosphärische Luft wurde in der üblichen Weise gewaschen und gehörig getrocknet in den Apparat geleitet. In den folgenden vier Tabellen (III-VI) finden sich die Abkühlungszeiten für dieses Gas in den zwei verschiedenen Hüllen und Bädern. Unter der Rubrik Bemerkungen finden sich die letzten Grundlagen der Berechnung, nämlich die constanten Zäbler $n . \log t_{0}-\Sigma\left(\log t_{y}\right)$ der Gleichung (II), $\S 5$, für die Ablühlungsgeschwindigkeit $v$, die Summe der Abkühlungszeiten $\Sigma\left(\vartheta_{v}\right)$; dann die $v_{t}$ und $v_{T}$ selber mit Angabe der berechneten $t$ und $T$; bei den $v_{t}$ und $v_{T}$ habe ich die Indices $t$ und $T$ weggelassen und sie nur mit $v_{1}$ und $v_{2}$ bezeichnet, wo die Indices auf die Hüllen sich beziehen. Es ergiebt sich also

1. für $k_{-59}:\left(v_{1}-v_{2}\right) \log e=0,000146$; und daraus nach Gleichung (I), $\S 5, k_{-59}$ selber zu

$$
k_{-59}=0,00003678 \frac{g}{\mathrm{~cm} \mathrm{sec}},
$$

2. für $k_{-149,5}:\left(v_{1}-v_{2}\right) \log e=0,0000800 ;$ und $k_{-149,5}$ selber wird

$$
k_{-149,5}=0,00002146 \frac{\mathrm{g}}{\mathrm{cm} \mathrm{sec}}
$$

3. aus diesen zwei Werten:

und

$$
k_{0}=0,00004677 \frac{\mathrm{g}}{\mathrm{em} \mathrm{sec}}
$$

$$
\gamma=0,00362
$$

ein Zahlenwert, wie ibn die Maxwell'sche Theorie verlangt. 





Wärmeleitungsfähigkeit der Gase.






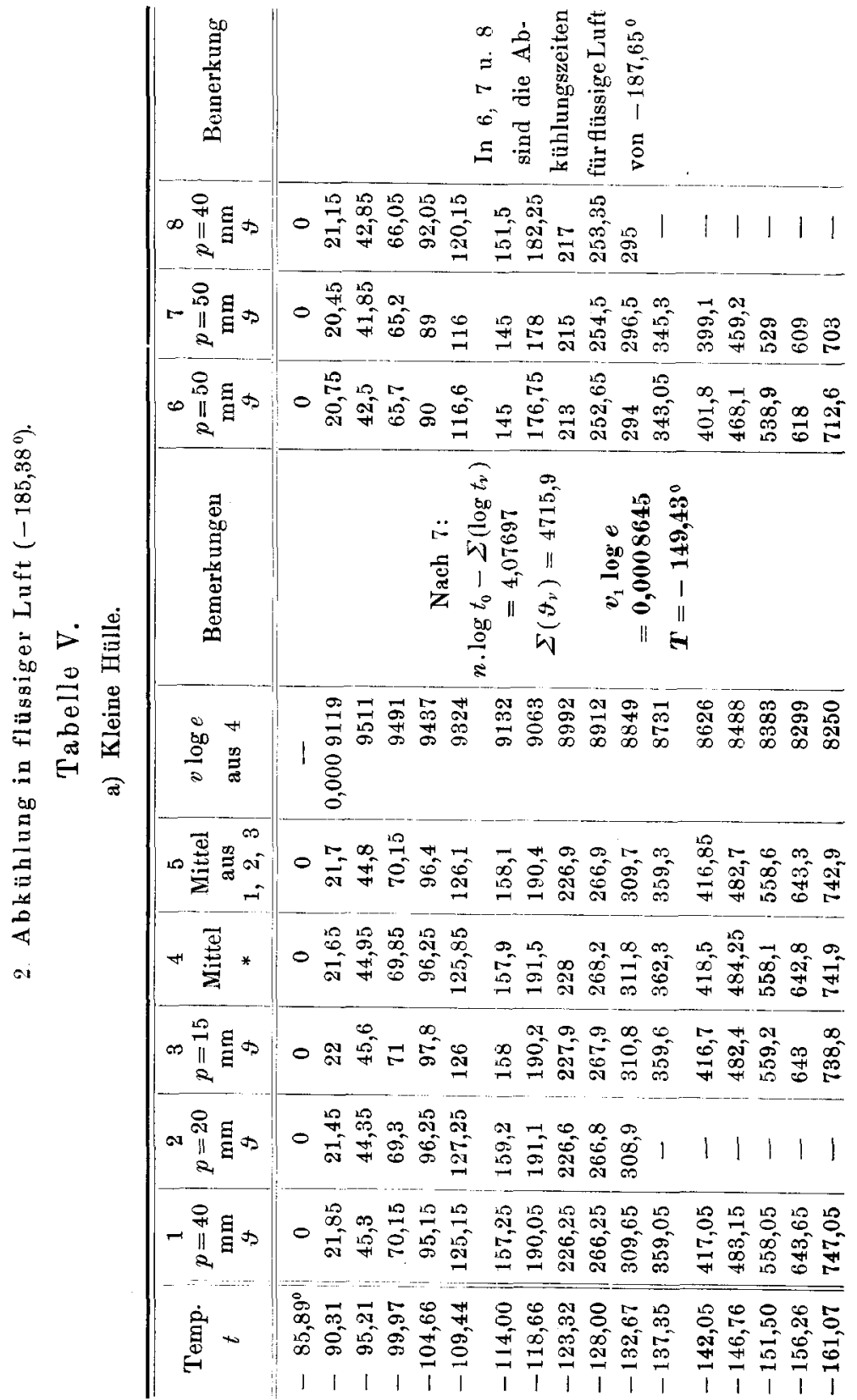


Wärmeleitungsfähigheit der Gase.

\begin{tabular}{|c|c|c|c|c|}
\hline & 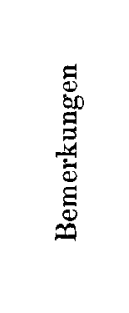 & & 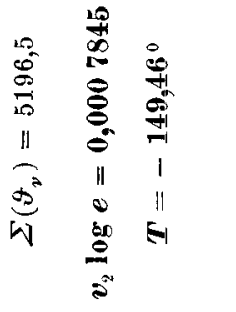 & \\
\hline & 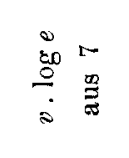 & 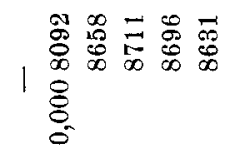 & 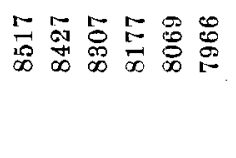 & 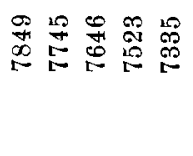 \\
\hline &  & 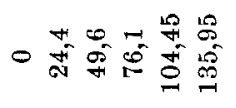 & 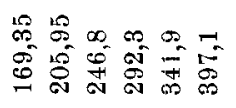 &  \\
\hline - & $\infty \|_{2}^{12} g$ & $0 \begin{array}{l}-\infty \\
\infty \\
\infty\end{array}$ & 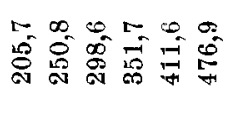 & 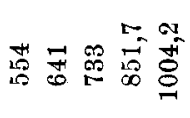 \\
\hline 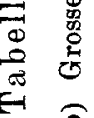 & 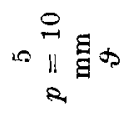 &  & 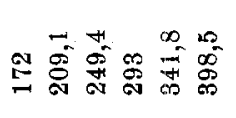 &  \\
\hline & $\begin{array}{c}8 \\
+11 \\
2\end{array}$ & 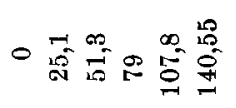 & 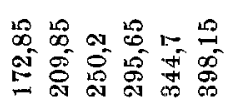 & 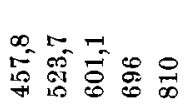 \\
\hline & $\infty{ }_{2}^{\infty} \mathbb{B}_{2}$ &  & 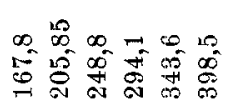 & 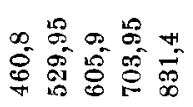 \\
\hline & 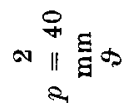 & 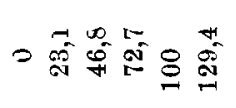 & 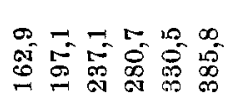 &  \\
\hline & 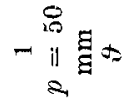 & 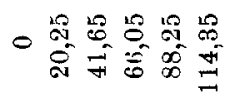 & 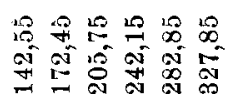 &  \\
\hline & $\underset{\dot{a}}{\dot{a}}+$ & 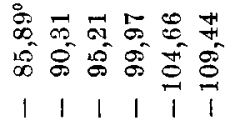 &  & 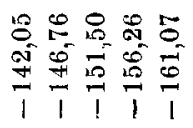 \\
\hline
\end{tabular}


II. Bestimmung der Leitungsfähigkeit $k$ für Wasserstoff und des Temperaturcoefficienten $\gamma$.

Das Gas wurde gewonnen aus chemisch reinem Zink und verdünnter Salzsäure, in concentrirter Kalilauge gewaschen, dann mit concentrirter Schwefelsäure (in einem Turm und in Trockenröhren mit Glasperlen), sowie mit Phosphorsäureanhydrid sorgfältig getrocknet. Die Beobachtungen mit dem ersten Kältebad gingen sehr gut von statten (vgl. Tab. VII und VIII). In flüssiger Luft jedoch machte sich folgende Störung bemerkbar. Wegen der raschen Abkühlung des Thermometers infolge der relativ grossen $W$ ärmeleitungsfähigkeit des Wasserstoffs trat bei mehreren Versuchen ein "Reissen“ des Thermometerfadens ein; die oberen Schichten des Petroläthers konnten den unteren, rasch sich zusammenziehenden Schichten im Stiel der Hülle nicht mehr folgen, es bildeten sich in dem untersten Teile der Capillare leere Blasen, welche bei der Erwärmung des Thermometers wieder zusammengingen. Die Cohäsion des Petroläthers konnte die Adhäsionskräfte am Glas nicht mehr überwinden. Dieses „Reissen" des Fadens trat ungefähr bei der Temperatur $-150^{\circ}$ und merkwürdigerweise bei der grösseren Hülle lieber ein als bei der kleineren, jedoch nicht bei allen Versuchen, sodass hier die Methode nicht versagte. Die unbrauchbaren Versuche wurden eliminirt, die Tabellen IX und $\mathrm{X}$ enthalten die verwendbaren.

Um zu zeigen, wie sich bei den Abkühlungsversuchen diese Störung geltend macht, habe ich in Tab. X, Columne 4 einen solchen Versuch vollständig angeführt. Bis zur Temperatur $-137^{\circ}$ (mit einem Strich bezeichnet) geht die Abkühlung sehr regelmässig vor sich, sodass sie die Werte Columne 3 bestätigt; von da ab scheint die Bildung der leeren Blasen zu beginnen, welche dann allmählich eine solche Verzögerung der Abkühlungszeiten herbeiführt, dass die schliessliche Differenz bis zu 35 Sec. (= 16 Proc.) anwächst.

Unter der Rubrik "Bemerkungen" finden sich in den folgenden Tabellen wieder die letzten Angaben, wie in den vorausgegangenen Tabellen, die Constanten $\left(n \log t_{0}-\Sigma\left(\log t_{v}\right)\right)$ sind nicht wiederholt, wie auch in den später folgenden 
Wärmeleitungsfähigkeit der Guse.

Tabellen. Auf Grund dieser Zahlen ergiebt sich also für Wasserstoff:

1. für $k_{-59,5}:\left(v_{1}-v_{2}\right) \log e=0,000950$; und daraus

$$
k_{-59}=0,0002393 \frac{\mathrm{g}}{\mathrm{em} \mathrm{sec}}
$$

2. für $k_{-150}:\left(v_{1}-v_{2}\right) \log e=0,000438$ und daraus

$$
k_{-150}=0,0001175 \frac{\mathrm{g}}{\mathrm{em} \mathrm{sec}}
$$

3. aus diesen zwei Werten:

$$
k_{0}=0,0003186 \frac{\mathrm{g}}{\mathrm{cm} \mathrm{sec}}
$$

und der Temperaturcoefficient $\gamma=\mathbf{0 , 0 0 4 2 2}$; ein Wert, der grösser ist als der von der Maxwell'schen Theorie geforderte und sich durch die geschilderten Störungen bei den Versuchen mit diesem Gase erklären lässt.

1. Abkühlung im Kältebad $\left(-80,58^{\circ}\right)$.

\begin{tabular}{|c|c|c|c|c|c|c|c|c|}
\hline $\begin{array}{c}\text { Tem- } \\
\text { peratur } \\
t\end{array}$ & $\begin{array}{c}1 \\
\text { Atmosph. } \\
\text { Druck } \\
\vartheta\end{array}$ & $\begin{array}{c}2 \\
p=50 \\
\mathrm{~mm} \\
\vartheta\end{array}$ & $\begin{array}{c}3 \\
p=30 \\
\mathrm{~mm} \\
y\end{array}$ & $\begin{array}{c}4 \\
p=10 \\
\mathrm{~mm} \\
\vartheta\end{array}$ & $\begin{array}{c}5 \\
\text { Mittel } \\
*\end{array}$ & $\begin{array}{c}6 \\
\text { Mittel } \\
\text { aus } \\
2,3,4\end{array}$ & $v \log e$ & Bemerk ungen \\
\hline$-16,22^{\circ}$ & 0 & 0 & 0 & 0 & 0 & 0 & - & \\
\hline$-21,54$ & 4,7 & 5,2 & 5,2 & 5,05 & 5,1 & 5,15 & 0,007347 & \\
\hline$-26,74$ & 9,7 & 10,5 & 11,1 & 10,7 & 10,7 & 10,8 & 7244 & \\
\hline$-31,82$ & 15,6 & 16,6 & 17 & 16,85 & 16,7 & 16,8 & 7222 & Nach 5: \\
\hline$-36,55$ & 22,5 & 23,75 & 23,9 & 24,1 & 23,5 & 23,9 & 7090 & $\Sigma\left(\vartheta_{v}\right)=559,5$ \\
\hline$-41,98$ & 29,85 & 31,85 & 32,25 & 32,2 & 31,6 & 32,1 & 7025 & \\
\hline$-47,02$ & 39,4 & 40,8 & 40,95 & 41,7 & 40,9 & 41,15 & 6914 & $v_{1} \log e=0.006698$ \\
\hline$-52,07$ & 49 & 51,25 & 51,9 & 51,9 & 51,5 & 51,7 & 6866 & $v_{1} 10 \mathrm{~g} e=0,000080$ \\
\hline$-56,90$ & 61,6 & 63,75 & 64,65 & 65,15 & 63,7 & 64,5 & 6817 & $t=-\overline{\mathbf{a}} 9,5^{\circ}$ \\
\hline$-61,80$ & 77,6 & 80,35 & 81,5 & 82 & 80,3 & 81,3 & 6661 & \\
\hline$-66,64$ & 98,8 & 101,4 & 102,8 & 104 & 101,5 & 102,7 & 6545 & \\
\hline$-71,51$ & 130,8 & 134,2 & 135,8 & 137 & 134 & 135,7 & 6354 & \\
\hline
\end{tabular}

Tabelle VII.

a) Kleine Hülle. 
Tabelle VIII.

b) Grosse Hülle.

\begin{tabular}{|c|c|c|c|c|c|c|c|}
\hline $\begin{array}{c}\text { Tem- } \\
\text { peratur } \\
t\end{array}$ & $\begin{array}{c}1 \\
p=50 \\
\operatorname{mm} \\
\vartheta\end{array}$ & $\begin{array}{c}2 \\
\substack{p=30 \\
\mathfrak{y}}\end{array}$ & $\begin{array}{c}3 \\
p=10 \\
\operatorname{mm} \\
\vartheta\end{array}$ & $\begin{array}{c}4 \\
\text { Mittel } \\
*\end{array}$ & $\begin{array}{c}5 \\
\text { Mittel } \\
\text { aus } \\
1,2,3\end{array}$ & $v \log e$ & Bemerkungen \\
\hline$-16,22^{\circ}$ & 0 & 0 & 0 & 0 & 0 & - & \\
\hline$-21,54$ & 5,9 & 6,1 & 6,1 & 6 & 6,05 & 0,006245 & \\
\hline$-26,74$ & 12,6 & 12,5 & 12,45 & 12,5 & 12,5 & 6201 & \\
\hline$-31,82$ & 19,9 & 19,9 & 20 & 19,9 & 19,9 & 6058 & Nach 4 \\
\hline$-36,55$ & 28 & 26,95 & 28,25 & 27,6 & 27,55 & 6034 & $\Sigma\left(y_{\nu}\right)=652,1$ \\
\hline$-41,98$ & 37,2 & 37,15 & 37,25 & 37,2 & 37,2 & 5960 & \\
\hline$-47,02$ & 48,4 & 47,65 & 47,8 & 47,8 & 47,95 & 5918 & $v_{2} \log e=0,005743$ \\
\hline$-52,07$ & 59,8 & 59,4 & 60 & 60,6 & 59,75 & 5835 & $t=-595^{\circ}$ \\
\hline$-56,90$ & 74,5 & 74,1 & 74,85 & 75,3 & 74,5 & 5634 & \\
\hline$-61,80$ & 92,2 & 93 & 93,1 & 93,8 & 93 & 5703 & \\
\hline$-66,64$ & 117 & 117,1 & 117,15 & 117,9 & 117,1 & 5635 & \\
\hline$-71,51$ & 152,8 & 152,1 & 153,1 & 153,5 & 152,7 & 5544 & \\
\hline
\end{tabular}

2. Abkühlüng in flüssiger Luft $\left(-185,38^{\circ}\right)$.

Tabelle IX.

a) Kleine Hülle.

\begin{tabular}{|c|c|c|c|c|c|c|}
\hline $\begin{array}{c}\text { Tem- } \\
\text { peratur } \\
t\end{array}$ & $\begin{array}{c}1 \\
p=50 \\
\mathrm{~mm} \\
\mathscr{Y}\end{array}$ & $\begin{array}{c}2 \\
p=30 \\
\mathrm{~mm} \\
y\end{array}$ & $\begin{array}{c}3 \\
p=10 \\
\mathbf{m m} \\
y\end{array}$ & $\begin{array}{c}4 \\
\text { Mittel } \\
\text { aus } \\
2,3\end{array}$ & $v \log e$ & Bemerkungen \\
\hline$-85,89^{0}$ & 0 & 0 & 0 & 0 & - & \\
\hline$-90,31$ & 5,15 & 5,1 & 5,1 & 5,1 & 0,003871 & \\
\hline$-95,21$ & 10,6 & 10,55 & 10,9 & 10,7 & 3817 & \\
\hline$-99,97$ & 16,3 & 16,7 & 16,9 & 16,8 & 3922 & \\
\hline$-104,66$ & 22,3 & 22,8 & 23,2 & 23 & 3915 & \\
\hline$-109,44$ & 28,8 & 29,6 & 30,1 & 29,8 & 3898 & Nach 4 \\
\hline$-114,00$ & 35,9 & 37 & 38 & 37,5 & 3796 & 4180 \\
\hline$-118,66$ & 43,5 & 45,1 & 45,9 & 45,5 & 3781 & 148,8 \\
\hline$-123,32$ & 51,7 & 53,9 & 54,6 & 54,2 & 3755 & \\
\hline$-128,00$ & 60,8 & 64,2 & 64,6 & 64,4 & 3700 & $v_{1} \log e=0,003549$ \\
\hline$-132,67$ & 70,3 & 75,1 & 75,1 & 75,1 & 3669 & $T=-149,73^{0}$ \\
\hline$-137,35$ & 81,4 & 87,95 & 87,6 & 87,6 & 3627 & \\
\hline$-142,05$ & 93,5 & 101,7 & 101,2 & 101,2 & 3567 & \\
\hline$-146,76$ & - & 117,1 & 117 & 117 & 3512 & \\
\hline$-151,50$ & - & - & $13 \bar{\jmath}$ & 135 & 3466 & \\
\hline$-156,26$ & - & - & 157,9 & 157,9 & 3378 & \\
\hline$-161,07$ & - & - & $180^{\circ}$ & 186 & 3290 & \\
\hline
\end{tabular}


Tabelle X.

b) Grosse Hülle.

\begin{tabular}{|c|c|c|c|c|c|c|}
\hline $\begin{array}{c}\text { Tem- } \\
\text { peratur } \\
t\end{array}$ & $\begin{array}{c}1 \\
p=50 \\
\mathrm{~mm} \\
\vartheta\end{array}$ & $\begin{array}{c}2 \\
p=10 \\
\mathrm{~mm} \\
\vartheta\end{array}$ & $\begin{array}{c}\mathbf{3} \\
p=10 \\
\mathrm{~mm} \\
\boldsymbol{9}\end{array}$ & $\begin{array}{c}4 \\
\text { Mittel } \\
\text { aus } \\
2,3\end{array}$ & $v \log e$ & Bemerkungen \\
\hline$-85,89^{\prime}$ & 0 & 0 & 0 & 0 & - & \\
\hline$-90,31$ & 5,8 & 6 & 5,85 & 5,9 & 0,003291 & \\
\hline$-95,21$ & 12,1 & 12,2 & 12,15 & 122 & 3504 & \\
\hline$-99,97$ & $18, \tilde{5}$ & 19,2 & 19,1 & 19,15 & 3453 & \\
\hline$-104,66$ & 25,5 & 26,3 & 26,2 & 26,25 & 3455 & \\
\hline$-109,44$ & 33,2 & 34 & 34 & 34 & 3451 & Nach? \\
\hline$-114,00$ & 42,4 & 43 & 42,7 & 42,85 & 3354 & Nach 2 \\
\hline$-118,66$ & 50,6 & 52,2 & 51,9 & 52,05 & 3325 & $\Sigma\left(\mathscr{\theta}_{\nu}\right)=1310,1$ \\
\hline$-123,32$ & 60,5 & 62,6 & 62,1 & 62,35 & 3243 & \\
\hline$-128,00$ & 71,2 & 73,3 & 73,85 & 73,6 & 3260 & $v_{2} \log e=0,003111$ \\
\hline$-132,67$ & 82,9 & 86,1 & 86,75 & 86,4 & 3205 & $T=-150,2^{\circ}$ \\
\hline$-137,35$ & 96,3 & 100 & 102 & 101 & 3163 & \\
\hline$-142,05$ & 112,2 & 116 & $\overline{119}, 7$ & - & 3112 & \\
\hline$-146,76$ & $129, \bar{\partial}$ & 134,1 & 140,8 & - & 3065 & \\
\hline$-151,50$ & 149,3 & $155, \breve{5}$ & 165,8 & - & 3009 & \\
\hline$-156,26$ & $\mathbf{1 7 3 , 5}$ & 180 & 201 & - & 2964 & \\
\hline$-161,07$ & 203,5 & 209,6 & 244,9 & - & 2920 & \\
\hline
\end{tabular}

III. Bestimmung der Wärmeleitungsfähigkeit $k$ für Koblensäure und des Temperaturcoefficienten $\gamma$.

Das Gas wurde aus Marmor und verdünnter Salzsäure entwickelt, in einer gesättigten Lösung von doppeltkohlensaurem Natron gereinigt, durch und über concentrirte Schwefelsäure, durch Chlorcalcium und über Phosphorsäureanhydrid in den Apparat geführt. Versuche mit Kohlensäure wurden nur mit der Kältemischung aus Aether und fester Kohlensäure angestellt. Tab. XI und XII enthalten die Angaben. Die Abkühlungszeiten mussten hier entsprechend der geringen Wärmeleitungsfähigkeit der Kohlensäure viel grösser ausfallen, als bei den rorausgegangenen Versuchen.

Die Abkühlung erfolgte so langsam, dass bei der Bestimmung des Zeitmomentes für die abzulesende Einstellung namentlich bei den tieferen Temperaturen oft zwei Zeitpunkte 
Tabelle XI.

Abkühlung im Kältebad $\left(-80,58^{\circ}\right)$.

a) Kleine Hülle.

\begin{tabular}{|c|c|c|c|c|c|c|c|}
\hline $\begin{array}{c}\text { Tem- } \\
\text { peratur } \\
t\end{array}$ &  & $\begin{array}{c}2 \\
p=50 \\
\mathrm{~mm} \\
\vartheta\end{array}$ & $\begin{array}{c}3 \\
p=50 \\
\mathrm{~mm} \\
y\end{array}$ & $\begin{array}{c}4 \\
p=30 \\
\mathrm{~mm} \\
y\end{array}$ & $\begin{array}{c}5 \\
\text { Mittel }\end{array}$ & $v \log e$ & Bemerkungen \\
\hline$-16,22^{\circ}$ & 0 & 0 & 0 & 0 & 0 & - & \multirow{12}{*}{$\begin{array}{c}\Sigma\left(\vartheta_{v}\right)=3577,6 \\
v_{1} \log e=0,001046 \\
t=-59^{\circ}\end{array}$} \\
\hline$-21,54$ & 17,4 & 31,3 & 31 & 31,2 & 31,2 & 0,001201 & \\
\hline$-26,74$ & 37,3 & 65,2 & 65,9 & 65,7 & 65,6 & 1182 & \\
\hline$-31,82$ & 59,2 & 104,3 & 104,7 & 104,7 & 104,6 & 1152 & \\
\hline$-36,55$ & 83,4 & 147,5 & 148 & 148,4 & 148,2 & 1112 & \\
\hline$-41,98$ & 113 & 197,8 & 199 & 198,9 & 198,6 & 1119 & \\
\hline$-47,02$ & 147 & 257,7 & 258,5 & 257,1 & 257,8 & 1097 & \\
\hline$-52,07$ & 188,2 & 327,1 & 326,5 & 324,7 & 326,1 & 1084 & \\
\hline$-56,90$ & 238,9 & 413,5 & 409,6 & 407,6 & 410,2 & 1058 & \\
\hline$-61,80$ & 306,9 & 519,4 & 513,5 & 511,6 & 514,8 & 1039 & \\
\hline$-66,64$ & 401,2 & 659,4 & 652,4 & 652,9 & 654,9 & 1014 & \\
\hline$-71,51$ & 547,7 & 875,4 & 857 & 864,5 & 865,6 & 0983 & \\
\hline
\end{tabular}

Tabelle XII.

b) Grosse Hülle.

\begin{tabular}{|c|c|c|c|c|c|c|}
\hline $\begin{array}{c}\text { Tem- } \\
\text { peratur } \\
t\end{array}$ & $\begin{array}{c}1 \\
\text { Atmosph. } \\
\text { Druck } \\
\vartheta\end{array}$ & $\begin{array}{c}2 \\
p=30 \\
\mathrm{~mm} \\
\vartheta\end{array}$ & $\begin{array}{c}3 \\
p=10 \\
\mathrm{~mm} \\
\vartheta\end{array}$ & $\begin{array}{c}4 \\
\text { Mittel }\end{array}$ & $v \log e$ & Bemerkungen \\
\hline$-16,22^{\circ}$ & 0 & 0 & 0 & 0 & - & \\
\hline$-21,54$ & 17 & 32,5 & 36,4 & 34,4 & 0,001088 & \\
\hline$-26,74$ & 36,2 & 69,4 & 75,4 & 72,4 & 1070 & \\
\hline$-31,82$ & 57,2 & 111,5 & 120,4 & 115,4 & 1044 & \\
\hline$-36,55$ & 81,8 & 159,4 & 169,4 & 164,4 & 1014 & $\Sigma\left(\vartheta_{v}\right)=3978,6$ \\
\hline$-41,98$ & 110,9 & 215,7 & 228,2 & 221,8 & 1001 & \\
\hline$-47,02$ & 144,8 & 279,5 & 294,9 & 287,2 & 0985 & $v_{2} \log e=0,0009413$ \\
\hline$-52,07$ & 186,1 & 356 & 377,2 & 366,6 & 0964 & $\boldsymbol{t}=-\mathbf{5 9 ^ { \circ }}$ \\
\hline$-56,90$ & 238,7 & 453,9 & 471,4 & 462,6 & 0939 & \\
\hline$-61,80$ & 307 & 579,9 & 593,4 & 586,6 & 0912 & \\
\hline$-66,64$ & 404,2 & 337,9 & 749,3 & 743,6 & 0893 & \\
\hline$-71,51$ & 563,3 & $\left.987,3^{1}\right)$ & $\left.987,4^{1}\right)$ & 987,35 & 0864 & \\
\hline
\end{tabular}

1) Es ist selbstverständlich nur Zufall, dass die zwei letzten Zahlen in 2 und 3 nahezu einander gleich sind. 
Wärmeleitungsfähigkeit der Gase.

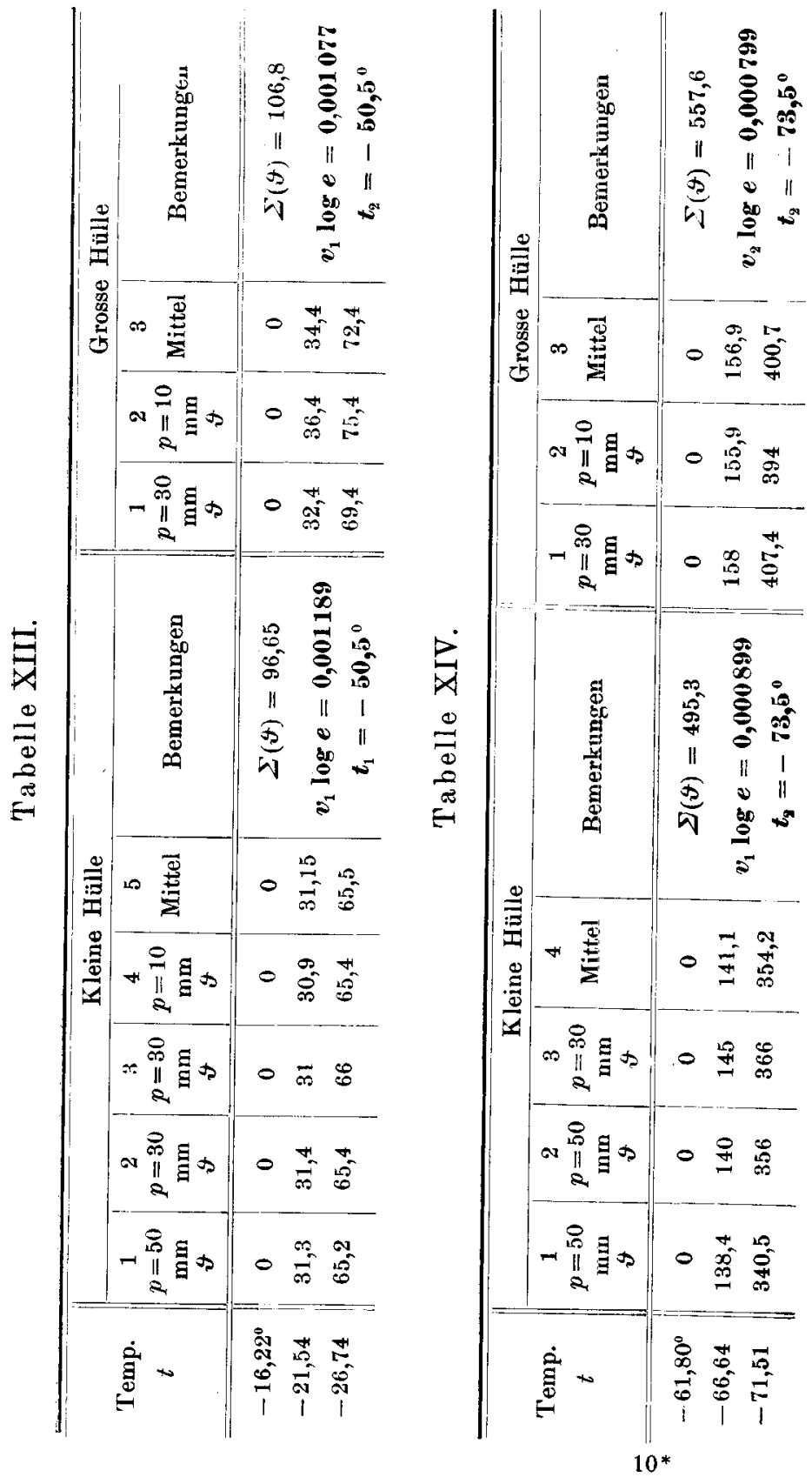


bestimmt werden konnten, die um mehrere Secunden auseinander lagen. Als zugehöriger Zeitpunkt für die Einstellung wurde die Mitte dieses kleinen Zeitintervalls genommen. So konnten die Beobachtungsfehler herabgesetzt werden. Die Versuche stimmen verhältnismässig gut überein. Die grösste Differenz, welche die zwei untersten Zahlen in Tab. XI, Columne 3 und 4 aufweisen, entspricht einer Abweichung von 2 Proc., fällt also noch ins Bereich der Beobachtungsfehler; indessen zeigen die zwei nächsten, höher gelegenen Zahlen nur mehr 1,7 proc., die folgenden 1,2 proc. Abweichung.

Von Tab. XII kommen die Versuche Columne 3 und 4 in Frage, sie weichen anfangs mehr voneinander $a b$, als es bei den tieferen Temperaturen der Fall ist.

Da die Versuche nur in dem einen Kältebad ausgeführt wurden, liesse sich wohl das $k$ für die mittlere Temperatur $t$, nicht aber das $\gamma$ berechnen, nun habe ich versucht, ob nicht $\gamma$ doch bestimmt werden könnte aus den Versuchen mit dem einen Kältebad. Es erstrecken sich die Abkühlungsversuche doch auf ein Temperaturintervall von über $55^{\circ}$; sind die beobachteten Werte von einiger Genauigkeit, so müssen sich doch zwei Mitteltemperaturen $t_{1}$ und $t_{2}$ und die diesen Temperaturen entsprechenden $v_{1}$ und $v_{2}$ bestimmen lassen, die das $\gamma$ nach der bisher angewendeten Methode berechnen lassen mit derselben Genauigkeit, mit welcher diese Grössen $t_{1}, t_{2}$ und $v_{1}$ und $v_{2}$ sich selber ergeben haben. Zu diesem Zwecke nehme ich als erstes Temperaturintervall aus dem grossen Intervall der Tab. XI und XII das von $-16,22^{\circ}$ bis $-26,74^{\circ}$; als zweites das von $-61,80^{\circ}$ bis $-71,51^{\circ}$. Die Tab. XIII und XIV enthalten die hierauf bezüglichen Grössen je für die kleine und grosse Hülle. Die $\boldsymbol{\Sigma}(\vartheta)$ sind aus den Mitteln genommen. Die $t_{1}$ und $t_{2}, v_{1}$ und $v_{2}$ sind berechnet ganz so, wie im Vorausgehenden.

Für die Mitteltemperaturen $t_{1}=-50,5^{\circ}$ und $t_{2}=-73,5^{0}$ waren die Wasserwerte noch zu bestimmen, deren Werte sich belaufen $\mathrm{zu}$

$$
C_{1}=0,1435
$$

und

$$
C_{2}=0.1450
$$


Auf Grund dieser Zahlen und der Werte in Tab. XIII und XIV ist

1. für $k_{-50,5}:\left(v_{1}-v_{2}\right) \log e=0,000112$ und hieraus

$$
\boldsymbol{k}_{-\mathbf{b} 0,5}=0,00002824 \frac{\mathrm{g}}{\mathrm{em} \mathrm{see}}
$$

2. für $k_{-73,5}:\left(v_{1}-v_{2}\right) \log e=0,000100$ und daher

$$
k_{-78,5}=0,00002546 \frac{\mathrm{g}}{\mathrm{em} \mathrm{se} e}
$$

3. aus diesen zwei Werten:

$$
k_{0}=0,00003434 \frac{\mathrm{g}}{\mathrm{cm} \mathrm{sec}}
$$

und $\gamma=\mathbf{0 , 0 0 3 5 2 ; ~ d e r ~ g e f u n d e n e ~ W e r t ~ f u ̈ r ~} \gamma$ ist auch bei Kohlensäure gleich dem von Maxwell geforderten.

Berechnet man aus Tab. XI und XII das $k_{-59}$ auf die gewöhnliche Weise und berechnet man das $k_{0}$ unter Voraussetzung des Maxwell'schen Temperaturcoefficienten, so erhält man die Zahlenwerte:

und

$$
k_{-59}=0,00002645
$$

$$
k_{\theta}=0,00003384,
$$

welche eine gute Uebereinstimmung erkennen lassen mit den Zahlengrössen, wie sie Tab. XIII und XIV ergeben haben.

\$ 6. Discussion der Resultate, ihre Stellung zur Theorie.

1. Absolute Wärmeleitungsfähigkeit.

Für atmosphärische Luft ergaben die Versuche:

und

$$
\boldsymbol{k}_{- \text {в9 }}=0,00003678
$$

$$
\boldsymbol{k}_{-150}=0,00002146 \text {. }
$$

Auf diese zwei Werte gründet sich

$$
k_{0}=0,0000467 \% \text {. }
$$

Die Theorie verlangt nach den neuesten Berechnungen ${ }^{1}$ ) für diese Grösse

$$
k_{0}=0,0000455 \text {. }
$$

1) O. E. Meyer, Theorie der Gase. p. 293. Breslau 1899. 
Die Uebereinstimmung des durch Beobachtung erhaltenen Wertes mit dem theoretisch geforderten wäre sehr gut; allein vergleicht man dieses $k_{0}$ mit den übrigen experimentell bestimmten, so ist er kleiner als alle anderen.

Früher fanden:

Kundt und Warburg $1875 k_{0}=0,000048$;

Winkelmann . . $1875 k_{0}=0,0000531$;

Graetz . . . . $1881 k_{0}=0,0000483$;

Winkelmann . . $1891 k_{0}=0,0000555$;

E. Müller . . . . $1896 k_{0}=0,000056 .^{1}$ )

Für Wasserstoff ergaben die Versuche die Werte

$$
\begin{aligned}
\boldsymbol{k}_{-59} & =0,0002393 ; \\
\boldsymbol{k}_{-150} & =0,0001175 .
\end{aligned}
$$

Aus diesen Werten ergiebt sich das $k_{0}$ für Wasserstoff zu

$$
k_{0}=0,0003186 .
$$

Im Gegensatze zu der gleichen Grösse $k_{0}$ für atmosphärische Luft zeigt diese Constante viel besseren Anschluss an früher bestimmte Werte.

Es fanden:

Kundt und Warburg $1875 k_{0}=0,000336$

Winkelmann . . . $1875 k_{0}=0,000336$

Graetz . . . . $1881 k^{0}=0,000316$

Winkelmann . . $1891 k_{0}=0,0003829$.

Für Kohlensäure gestaltete sich die Berechnung der Versuche etwas anders, wie schon angegeben, weil ich aus den Abkühlungsversuchen mit einem Kältebad auch die Abhängigkeit der Wärmeleitung dieses Gases von der Temperatur ermitteln wollte. Gebe ich zunächst den für die erste Mitteltemperatur $-59^{\circ}$ bestimmten Wert, so ist

$$
\boldsymbol{k}_{-59}=0,00002645 \text {. }
$$

1) Vgl. Näheres die Dissertationsschrift p. 49 u. 50. Ich habe $k_{0}$ für atmosphürische Luft auch mit einem Quecksilberapparat bestimmt und unter Annahme der Maxwell'schen Theorie: 0,00004791 gefunden, ein Wert, welcher den obigen, mit dem Petrolätherapparat gefundenen nahezu bestätigt. 
Berechne ich daraus unter Annahme des Maxwell'schen Gesetzes (für Kohlensäure ist seine Gültigkeit so ziemlich sicher) das $k_{0}$, so erhalte ich für $k_{0}$

$$
\boldsymbol{k}_{0}=0,00003384 ; \ldots \alpha \text {. }
$$

Nun wollte ich $k_{0}$ aus den Versuchen selber berechnen. Aus den zwei Werten

und

$$
\boldsymbol{k}_{-50, \tilde{a}}=0,00002824
$$

ergiebt sich

$$
k_{-73, \tilde{j}}=0,00002546
$$

$$
\boldsymbol{k}_{0}=0,00003434 ; \ldots \beta .
$$

Die zwei Werte von $k_{0}$ zeigen gute Uebereinstimmung. Der Wert sub $\beta$ schliesst sich dem Winkelmann'schen Resultat am engsten an.

Es fanden nämlich:

$$
\begin{aligned}
& \text { Winkelmann . } 1875 k_{0}=0,0000355 \\
& \text { Graetz . . . . } 1881 k_{0}=0,00003091 \\
& \text { Winkelmann . . } 1891 k_{0}=0,0000327 \text {. }
\end{aligned}
$$

2. Relative Wärmeleitungsfähigkeit.

Sie berechnet sich unabhängig von den Dimensionen der angewendeten Apparate aus den Differenzen der betreffenden Abkühlungsgeschwindigkeiten:

Wasserstoff $\left(k_{v}\right)$ und atmosphärische Luft $\left(k_{l}\right)$ miteinander verglichen giebt

$$
\begin{aligned}
& \text { 1. für } 0^{0}: \quad \frac{k_{w}}{k_{l}}=\mathbf{6}, 8 ; \\
& \text { 2. für }-59^{0}: \frac{k_{w}}{k_{l}}=\mathbf{6}, 6 ; \\
& \text { 3. für }-150^{0}: \frac{k_{w}}{k_{l}}=5,6 .
\end{aligned}
$$

Die Theorie verlangt für dieses Verhältnis 7,1, welches durch die zwei ersten Werte annähernd bestätigt wird.

Vergleicht man schliesslich noch die durch die Versuche erhaltenen Werte mit den theoretisch berechneten Grössen, und setzt man in beiden für Luft $k_{0}=1$, so erhält man als Resultat:

$\begin{array}{ccc} & k_{0} \text { beobachtet } & k_{0} \text { berechnet } \\ \text { für atmosphärische Luft } & 1 & 1 \\ \text { " Wasserstoff } & 6,8 & 7,1 \\ \text { " Kohlensäure } & 0,73 & 0,70 .\end{array}$


3. Die Temperaturcoefficienten.

Das meiste Interesse an den experimentellen Untersuchungen über die Wärmeleitungsfähigkeit der Gase nimmt der Temperaturcoefficient für sich in Anspruch, er ist auch für die Gastheorie das Wichtigste.

Die Versuche mit dem Petrolätherapparat ergaben:

1. für atmosphärische Luft $\gamma=0,00362$;

2. "Wasserstoff $\gamma=0,00422$

3. " Kohlensäure $\quad \gamma=0,00352$

und würden daher aussagen:

„Es ändert sich die Wärmeleitungsfähigkeit der atmosphärischen Luft innerhalb des Temperaturintervalls von $0^{\circ}$ bis - $180^{\circ}$ mit der Temperatur ausgesprochen im Sinne der Maxwell'schen Theorie, d. h. proportional der absoluten Temperatur selbst; in einiger Annäherung ist dasselbe auch der Fall für Wasserstoff; die Versuche mit Kohlensäure innerhalb des Intervalls von $0^{\circ}$ bis $-80^{\circ}$ bestätigen gleichfalls entschieden die Maxwell'sche Theorie für dieses Gas."

Was nun zunächst atmosphärische Luft und Wasserstoff anlangt, hat Graetz schon im Jahre 1881 durch seine Versuche gefunden, dass diese zwei Gase ihre Wärmeleitung mit der Temperatur ändern, wie es die Clausius'sche Theorie verlangt. Zehn Jahre später hat Winkelmann ${ }^{1}$ ) nach früher anderen, zur Maxwell'schen Theorie hinneigenden Werten die gleichen Resultate erhalten. ${ }^{2}$ )

Diese wie überhaupt alle früheren Bestimmungen der Temperaturcoefficienten sind hergeleitet aus dem Temperaturbereich von $0^{\circ}$ bis $+100^{\circ}$ bez. $+200^{\circ}$.

Für Temperaturen oberhalb des Eispunktes würden also die früheren Versuche zum grossen Teil Clausius'sche Temperaturcoefficienten für die zwei Gase erfordern, und für negative Temperaturen ergeben vorliegende Versuche für diese Grössen den Maxwell'schen Wert.

Sind nun die früheren und die gegenwärtigen Resultate miteinander vergleichbar, um aus ihnen für die einander

1) Winkelmann, Wied. Ann. 44. p. 177. 1891.

2) Ueber weitere Resultate vgl, noch Graetz, Wied. Ann. 45. p. 298. 1892, wo die Zusammenstellung gegeben ist. 
gegenüberstehenden Theorien überhaupt Folgerungen zu ziehen? Sie können dann miteinander verglichen werden, wenn bei ihnien die absoluten Wärmeleitungsconstanten $k_{0}$ für die untersuchten Gase einander verhältnismässig nahe kommen. Nun ist bereits (vgl. diesen Paragraph, Abschnitt 1) erwähnt, dass die $k_{0}$ für Wasserstoff und Kohlensäure ganz gut übereinstimmen, das $k^{0}$ für atmosphärische Luft zeigt insofern eine Abweichung von den früheren Werten, als es kleiner ist, als alle früher erhaltenen, dafür kommt es allerdings auch dem theoretisch geforderten Werte näher als alle übrigen, die $\mathrm{Ab}$ weichung ist nicht grösser, als es Beobachtungsfehler bewirken können, wie eine nähere Prüfung zeigt.

Angenommen, es kommen die beobachteten Werte für die Temperaturcoëfficienten bei den verschiedenen Temperaturintervallen ober- und unterhalb des Eispunktes wirklich den wahren Werten sehr nahe, so würde für die Theorie etwa folgen:

Die vorliegenden Untersuchungen lassen erkennen, dass die Wärmeleitungsfähigkeit der zwei Gase Luft und Wasserstoff durchaus nicht so weiter über den Eispunkt herab durch den negativen Temperaturbereich hindurch mit der Temperatur sich ändert, wie die Clausius'sche Theorie dies verlangt, dass sie vielmehr nach unten hin bei tiefen Temperaturen rascher abnimmt, als diese Theorie es erfordert; oder umgekehrt würde das lauten: Die Wärmeleitungsfähigkeit dieser zwei Gase scheint rom absoluten Nullpunkt an durch die tieferen Temperaturen hindurch mit der Temperatur zu wachsen im Sinne der Maxwell'schen Theorie, nimmt aber nicht so weiter durch den Nullpunkt (Eispunkt) hindurch im Bereich der positiven Temperaturen zu, sondern diese Aenderung nimmt mit wachsender Temperatur (positiver) allmählich ab.

Das hiesse aber eigentlich nichts anderes als: die Aenderung der Wärmeleitung dieser Gase mit der Temperatur scheint überhaupt nicht so einfach linear vor sich zu gehen, wie die beiden Theorien es erfordern; möglicherweise sind es moleculare Veränderungen im Gase (Association der Molecüle bei tiefen, Dissociation derselben bei höheren Temperaturen, radiale Veränderungen der Moleküle), welche die einfache Aenderung, wie sie die Theorie verlangt, zu einer komplicirteren gestalten.

Das wäre selbstverständlich nur eine Vermittelung der 
154 P. A. Eckerlein. Wärmeleitungsfähigkeit der Gase.

früheren, experimentell erhaltenen Resultate mit den Ergebnissen dieser Untersuchung. Dass diese zu Gunsten der Maxwell'schen Theorie sprechen und zwar einmütig, mehr lässt sich für jetzt ohne weitere Beobachtungen nicht aussagen. Dass die Methode der Versuche sich bewährt hat, haben die absoluten Wärmeleitungsconstanten $k_{0}$, die sich aus den Beobachtungen ergeben haben, erkennen lassen.

Bezüglich des Temperaturcoefficienten der Kohlensäure sei schliesslich noch bemerkt, dass der aus dem Beobachtungsintervall von $0^{\circ}$ bis $-80^{\circ}$ resultirende Maxwell'sche Wert mit den früher beobachteten Werten gut übereinstimmt und die Gültigkeit der Maxwell'schen Theorie für dieses Gas auch für tiefe Temperaturen bestätigt.

Erlaube mir, Hrn. Prof. Graetz für die gütige Anregung zu dieser Arbeit und die freundliche Leitung derselben auch an dieser Stelle meinen ehrerbietigsten Dank auszusprechen.

München, Physik. Inst. d. kgl. Universität, 1900.

(Eingegangen 18. Juli 1900.) 